\title{
A New, Compact MR-Compatible Surgical Manipulator for Minimally Invasive Liver Surgery
}

\author{
Daeyoung Kim ${ }^{1}$, Etsuko Kobayashi ${ }^{2}$, \\ Takeyoshi Dohi ${ }^{1}$, and Ichiro Sakuma ${ }^{2}$ \\ ${ }^{1}$ Department of Mechano-Informatics, Graduate School of Information Science \\ \{young, etsuko\}@miki.pe.u-tokyo.ac.jp \\ ${ }^{2}$ Institute of Environment Studies, Graduate School of Frontier Sciences, \\ The University of Tokyo, 7-3-1 Hongo Bunkyo-Ku, Tokyo, 113-8656, Japan \\ $\{$ dohi, sakuma\}@miki.pe.u-tokyo.ac.jp
}

\begin{abstract}
Recently, intra-operative magnetic resonance imaging (IO-MRI) has become an important clinical procedure. To perform this work, MR-compatible surgical equipment is necessary. In this study, our research team developed the technology for a MR-compatible manipulator for minimally invasive liver surgery that can operate in an MRI-scanner without any significant influence on MR imaging. The developed system that allows for 6 Degrees of Freedom (D.O.F) and a new RCM mechanism and bending forceps actuated by a motorhydraulic actuation system. This manipulator provides enough power for liver surgery and is small enough to be operated within $45 \mathrm{~cm}$-high space in an OpenMRI. The image distortion within the MRI is minimal considering the effect of materials used in the system. These results demonstrate that it is feasible to operate the manipulator during MR operation and scanning.
\end{abstract}

\section{Introduction}

Recently, Intra-operative Magnetic Resonance Imaging (IO-MRI) has become a significant clinical procedure for minimally invasive surgery [1]-[4]. MR imaging is especially suitable for the intra-operative use because it does not require X-ray irradiation, allows for high soft-tissue contrast, and it can provide an arbitrary cross sectional image required for surgical planning. 3D-MR Angiograghy (3D-MRA) also provides information about blood vessel location. Surgeons can prevent unexpected bleeding during operation using this information.

However, space for the surgical operation within the MRI is still too limited for surgeons to perform conventional surgical work. Thus a mechanical manipulator that can perform precise surgical procedures in the limited space in open MRI is highly desirable. Furthermore, a MR image guided control of the manipulator can realize a new safer surgical procedure.

When we consider the application of an intra-operative MRI on minimally invasive liver surgery, the manipulator must be able to generate a large amount of power required for surgery. The simultaneous uses of multiple manipulators are often required, as demonstrated in the da Vinci system. In addition, since the liver is a soft organ, it is also necessary to conduct MR images during manipulator operation. 
Non-ferromagnetic materials and ultrasonic motors are widely used today to realize MR-compatible manipulators. However, noise from ultrasonic motors will cause significant influence on MR imaging when such motors are placed near the Field of View (FOV). When the actuator was placed far from the FOV, the resulting mechanical system would be overly large. Thus, it would be difficult to install in the limited space such as an open MRI system.

In this study, we outline a newly developed MR-compatible manipulator for minimally invasive liver surgery. New materials, a new actuation system, and a manipulator mechanism were developed and incorporated in the design of this MRcompatible manipulator. The result is a tool that allows for minimally invasive liver surgery that can be operated in an MRI-scanner without any significant influence on MR imaging. We evaluated image distortion by several non-ferromagnetic materials and optimized their usage and propose a new hydraulic actuation system that provides enough power for actuation while the ultrasonic motor-based power generators are placed away from the FOV of the MRI scanner. A new remote center of motion (RCM) mechanism is also proposed to miniaturize the manipulator system. In numerous tests and experiments, we could evaluate the MR-compatibility of the system.

\section{Manipulator Design}

\subsection{Material Selection}

Special materials are required for the making of the MR-compatible device. For the material's MR-compatibility, Chinzei [3] evaluated the material's effect on the MRI by testing with $2 \mathrm{~cm}$ Cubic metals, and Osada [5] analyzed the effect using the Finite Element Method (FEM). However, there is little data on the influence of material for practical mechanical structures used as surgical tools and manipulators. There is only a limited amount of quantitative data regarding the influenced area. In evaluating the effect of material on the MRI we used test pieces in the shape of pipe and rod that simulated the mechanical structure used in forceps.

For rods with a diameter of $10 \mathrm{~mm}$, the differences between observed MR images and the actual diameter was $10 \%$ of the actual diameter for $\mathrm{Be}-\mathrm{Cu}$ and copper, $300 \%$ for titanium, and more than $1900 \%$ for SUS304, and SUS316 respectively. For titanium pipe with diameter of $10 \mathrm{~mm}$, the differences between observed MR images and the actual diameter was $70 \%$ of the actual diameter. SUS304, and SUS316 pipe showed much larger distortion (more than 400\%). As for the test pieces with diameter of $3 \mathrm{~mm}$ (both for pipes and rods), there is very little distortion for $\mathrm{Be}-\mathrm{Cu}$, copper and titanium. There was significant distortion for SUS304, and SUS316 pipes. For $10 \mathrm{~mm}$ rods, range of influenced area was $10 \mathrm{~mm}$ from the test piece for $\mathrm{Be}-\mathrm{Cu}$ and copper, $30 \mathrm{~mm}$ for titanium, and 300mm for SUS304 and SUS316. Therefore, it is appropriate to use $\mathrm{Be}-\mathrm{Cu}$ for forceps that is placed near the center of FOV. Titanium pipe can be used for mechanical structure placed far from FOV by $30 \mathrm{~mm}$.

Since another mechanical part, such as a spring, might be used in manipulator mechanism, we also conducted the same evaluation on spring made of SUS 306, Be$\mathrm{Cu}$ and NAS106N. The results are shown in Table 1. 
Table 1. Evaluation of springs

\begin{tabular}{|c||c|c|c|}
\hline Material of spring & SUS306 & Be-Cu & NAS106N \\
\hline \hline Diameter of wire (mm) & 0.3 & 0.3 & 0.3 \\
\hline Inner diameter of spring (mm) & 0.9 & 0.9 & 1.3 \\
\hline Outside diameter of spring (mm) & 1.5 & 1.5 & 1.9 \\
\hline Spring constant (gf/mm) & 30 & 7 & 500 \\
\hline $\begin{array}{c}\text { Observed image size evaluated two } \\
\text { perpendicular diameter (mm) }\end{array}$ & $58.5 \times 94.5$ & $1.8 \times 1.8$ & $7.0 \times 11.0$ \\
\hline \begin{tabular}{c} 
Range of influenced area (mm) \\
\hline
\end{tabular} & 100 & $\leq 10$ & $\leq 10$ \\
\hline
\end{tabular}

As a result of these studies, $\mathrm{Be}-\mathrm{Cu}$ was selected for the tip, and aluminum, brass, titanium for the structure. Only NAS106 N used in the metal of stainless for the pin, the spring, etc. considering hardness.

\subsection{Actuation System}

We used a hydraulic driving system both for forceps and forceps positioning manipulator to remove influence of the system on MR imaging even when the manipulator system was being driven during scanning. Ultrasonic motors placed far from RF coils of the MR scanner generated hydraulic power. It was transmitted with small tubes filled with sterilized saline. Thus the sterilization of the actuators is possible.

In a conventional hydraulic system, various mechanical elements, such as pressure source, accumulator, and valves are requiring leading to an increase in system size. We used hydraulic bilateral cylinder to improve the controllability and to reduce the size of the actuator [8].

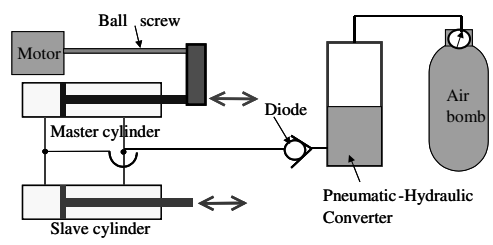

Fig. 1. Motor-hydraulic system

The system configuration of actuation system is shown in Fig.1. The torque of an ultrasonic motor controls the level of water pressure using a ball screw mechanism. Linear motion of the master cylinder is transmitted to the slave cylinder as shown in Fig.1. Leakage of fluid and immixture of air are two inherent problems in this system. To solve them, we added a fluid leakage compensation mechanism consisting of a compact air bomb, a pneumatic-hydraulic converter, and a hydraulic diode. This mechanism refills fluid when fluid pressure in the actuator cylinder is lower than a 
certain level as shown in Figure 1. We can reduce the effect of air bubbles by setting the fluid pressure in the cylinder to high.

\subsection{System Requirement}

The mechanism of the manipulator was designed to consider the use of the system in a commercially available Open-MRI scanner (AIRIS2000, Hitachi Medical Co.). Detailed requirements for the manipulator system is summarized as follows:

- The system should have 6 degrees of freedom for $\times 15 \mathrm{~cm} \times 6 \mathrm{~cm}$ size under $10 \mathrm{~cm}$ of abdominal wall considering the positioning and orientation of forceps in the abdominal space.

- The Range of motion of the forceps in the abdominal cavity is $25 \mathrm{~cm}$ size of liver and working space created under pneumoperitoneum.

- The system should be placed inside the $45 \mathrm{~cm}$ high opening space of open MRI scanner.

- The system should generate $4 \mathrm{kgf}-\mathrm{cm}$ torque around insertion port placed on the abdominal wall. This value was obtained by animal experiment with a pig that has $3 \mathrm{~cm}$-thickness abdominal wall.

The forceps should be able to bear $600 \mathrm{gf}$ considering the weight of liver tissue.

\subsection{System Design}

Since there was a restriction in height, the forceps should be inserted to the abdominal cavity from horizontal direction and should approach to the liver from vertical direction. Thus the bending of the forceps is indispensable.

In this system, bending was realized using a linkage mechanism. The length of the forceps tip (gripper) was $82 \mathrm{~mm}$ and the remote center of motion was set at the point of $22 \mathrm{~cm}$ from the tip of the forceps using the forceps positioning mechanism as described in section 2.5. The rotation of the gripper was achieved by cam mechanism. This mechanism enables the gripper to handle tissues such as a tubular blood vessel from any direction. Slave cylinders placed at the base of the forceps actuated the linkage mechanism and cam. Master cylinders were actuated by ball screw (lead: $2 \mathrm{~mm}$ ) driven by ultrasonic motors USR30-S4 (SHINSEI, Torque: $0.05 \mathrm{Nm}$ ). Lengths of tubes connecting master and slave cylinder was $150 \mathrm{~cm}$ and its inner diameter was $1.5 \mathrm{~mm}$.

In minimally invasive abdominal surgery, the Remote Center of Motion (RCM) of forceps is desirable where the forceps rotates around the insertion point on the abdominal wall [9]. We have developed a new RCM mechanism as shown in Figure 2.

To make RCM in this system, a ratio of $d_{t} / d_{b}$ must be fixed during entire motion of the mechanism. This is obtained by fixing the ratio of two linkages' linear motion $\mathrm{S}_{\mathrm{t}} / \mathrm{S}_{\mathrm{b}}$. Since the motion of two master cylinders determines $\mathrm{St} / \mathrm{Sb}$, the distance between the lower linkage and remote center $d_{b}$ was $5 \mathrm{~cm}$.

The total movement of the RCM can be achieved by setting the gear ratio in ball screw driving mechanism. Ball screws (lead: 10mm) were driven by an ultrasonic motor USR60-S4 (SHINSEI. torque: 0.5Nm). Lengths of tubes connecting master and 
slave cylinder was $150 \mathrm{~cm}$ and its inner diameter was $1.5 \mathrm{~mm}$. This RCM has sufficient power (4kgf-cm at least), while the system is compact enough to install in the narrow space in open MRI scanner $(9 \mathrm{~cm} \times 14 \mathrm{~cm} \times 45 \mathrm{~cm}$ in height $)$. The total system is shown in Fig.3.
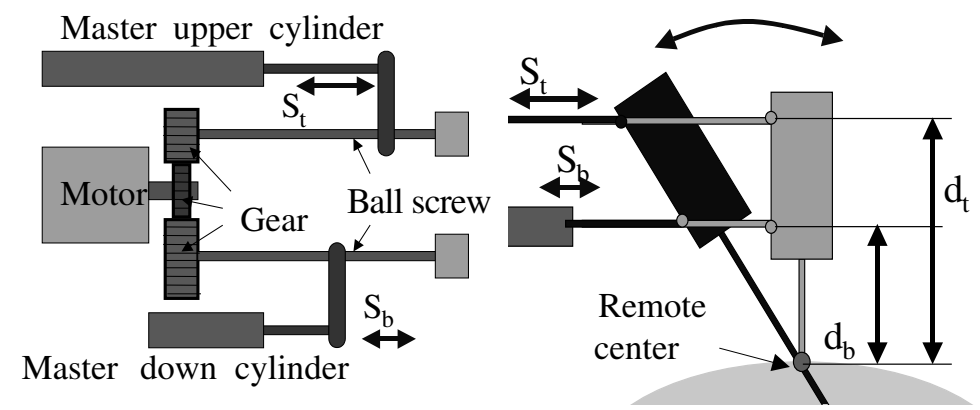

Fig. 2. RCM with linear actuators

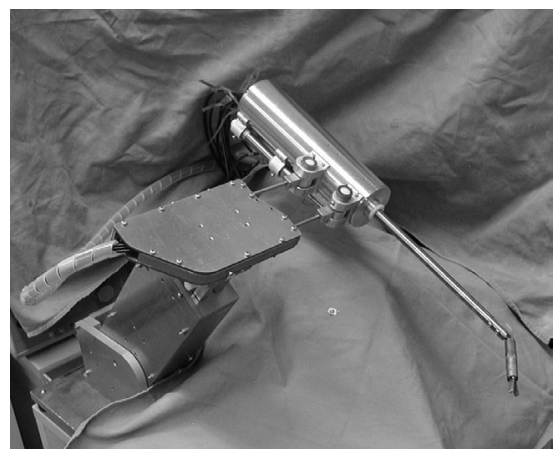

Fig. 3. MR-compatible Surgical Robot

\section{Evaluation Experiments and Results}

We evaluated the MR-compatibility of the system by placing and operating it in a conventional MR scanner (MRH-500 HITACHI Medical Co.). MR images were obtained and their distortions evaluated.

\subsection{MR-Compatibility of Forceps' Material}

Water and forceps were placed into acrylic plastic pipes of different diameters $(\phi 22 \mathrm{~mm}, \phi 58 \mathrm{~mm}, \phi 107 \mathrm{~mm})$. The range of influenced area by the forceps was measured (Fig. 4). Sequence was SE, TE/TR: 30/300, FOV: 256mm $\times 256 \mathrm{~mm}$, slice thickness: $10 \mathrm{~mm}$, and flip angle is 90 degrees. The results are summarized in Fig.4. We found that $\mathrm{t}$ distortions were small and limited in the area with a $10 \mathrm{~mm}$ radius. 

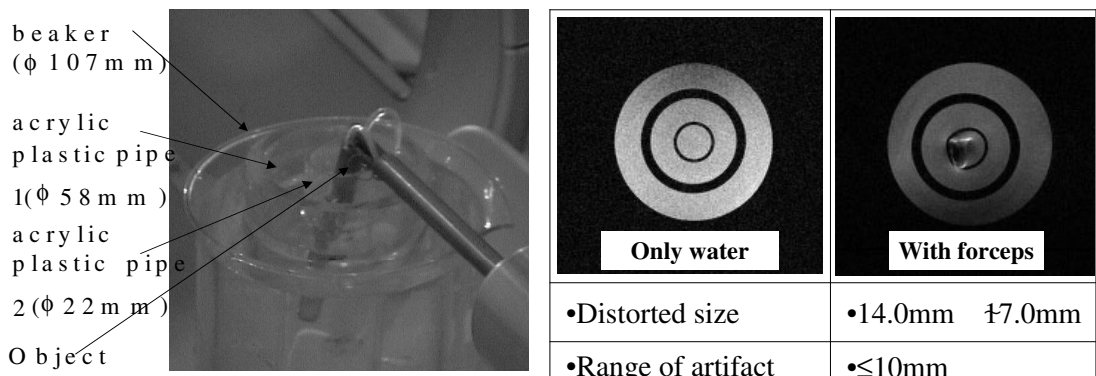

\begin{tabular}{|l|l|}
\hline$\bullet$ Distorted size & $\bullet 14.0 \mathrm{~mm} \square+7.0 \mathrm{~mm}$ \\
\hline$\cdot$ Range of artifact & $\bullet \leq 10 \mathrm{~mm}$ \\
\hline$\bullet$ Shift of material & $\bullet \leq 2 \mathrm{~mm}$ \\
\hline$\cdot$ S/N ratio:77.0 & $\bullet 78.8$ \\
\hline
\end{tabular}

Fig. 4. Evaluation :MR-compatibility of forceps' material

\subsection{MR-Compatibility of Motor-Hydraulic Driving}

We evaluated MR-compatibility of motor-hydraulic driving systems in the following four steps:

1. The manipulator was placed outside of RF coil, and electric power was unplugged.

2. The manipulator was placed inside of RF coil, and electric power was unplugged.

3. The manipulator was placed inside of RF coil, and electric power was plugged but did not drive the manipulator.

4. The manipulator was placed inside of RF coil, and electric power was plugged and drove the manipulator.
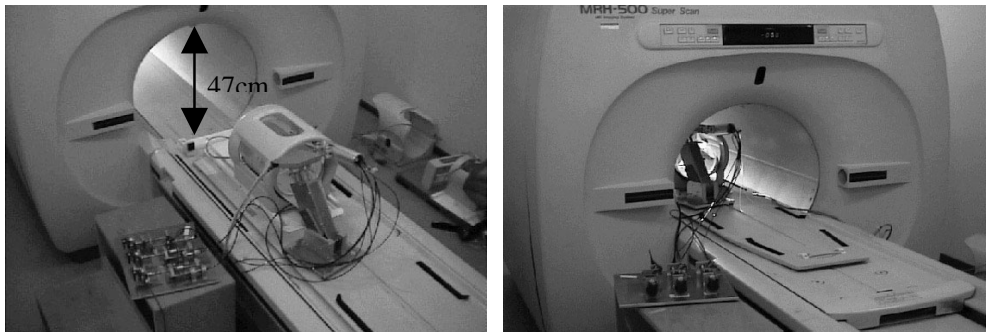

Fig. 5. Setting for evaluation: MR-compatibility of motor-hydraulic driving

Table 2. MR-compatibility of motor-hydraulic driving

\begin{tabular}{|c|c|c|c|c|}
\hline Condition & $\begin{array}{c}\text { Outside of RF } \\
\text { coil } \\
\text { Unplugged }\end{array}$ & $\begin{array}{c}\text { Inside of RF coil } \\
\text { Unplugged }\end{array}$ & $\begin{array}{c}\text { Inside of RF coil } \\
\text { Plugged }\end{array}$ & $\begin{array}{c}\text { Inside of RF coil } \\
\text { Being driven }\end{array}$ \\
\hline Image & & & & \\
& & & & \\
\hline S/N ratio & 283.7 & 275.6 & 261.1 & \\
\hline
\end{tabular}




\subsection{MR-Compatibility of the Total System}

This evaluation was completed through two steps with a phantom used in 5.1 evaluations.

1. The manipulator was placed in the phantom unit to measure the distortion of the shape of the mechanism in the MRI, but was not actuated. Electric power was supplied.

2. The manipulator was placed in the phantom unit and was actuated.

The $8 \mathrm{~cm}$ long tip of the forceps (gripper) was placed in the phantom. In step 2, bending mechanism was actuated. Results are shown in Table 3. Actuation of the manipulator slightly deteriorates image distortion and $\mathrm{S} / \mathrm{N}$ ratio.

Table 3. MR-compatibility of total system

\begin{tabular}{|c|c|c|}
\hline & Just plugged & With actuation \\
\cline { 2 - 3 } & \multicolumn{3}{|c|}{$\begin{array}{c}\text { Forceps were put } \\
\text { Into the phantom }\end{array}$} & & \\
& & \\
\hline Distorted size & $14 \times 17(\mathrm{~mm})$ & $15 \times 19.5(\mathrm{~mm})$ \\
\hline Range of artifact & $\leq 10 \mathrm{~mm}$ & $\leq 10 \mathrm{~mm}$ \\
\hline S/N ratio & 77.3 & 45.1 \\
\hline
\end{tabular}

\section{Discussion, Conclusion and Future Work}

We developed a MR-compatible Surgical Manipulator System with Hydraulic Actuators. The driving method using bilateral cylinders was powerful effective enough to realize MR-compatibility, making it possible to produce a compact surgical manipulator. No significant influence on MR imaging was observed even when the mechanism was actuated during scanning. By adding a leakage compensation mechanism, stability and controllability of the system were improved.

As for manipulator design, we developed a bending forceps system that can be inserted into the abdominal cavity from a horizontal direction that allows liver to be approached from a vertical direction. The bending and rotation were realized by a linkage and cam mechanism driven by hydraulic actuators. A new RCM using linear actuators was also proposed. The developed system has 6 D.O.Fs. It is compact in size to be installed in narrow space in the scanner $(45 \mathrm{~cm}$-high) and to be operated freely. The system has enough power (at least $4 \mathrm{kgf}-\mathrm{cm}$ ), and $600 \mathrm{gf}$ for holding the tissue while maintaining its compacted ness. It has the possibilities for enhanced safety [10] during usage as a medical robot.

Although the average $\mathrm{S} / \mathrm{N}$ ratio of $\mathrm{MR}$ images deteriorated when the total manipulator system was actuated, the range of influenced area was limited to $10 \mathrm{~mm}$ in radius and there no significant noise on image was obtained. The image distortion was minimal considering the effect of materials used in the system. These results demonstrated that it is feasible to operate the manipulator during MR scanning. 
Control of the surgical manipulator using real time image data of the important anatomical structure such as blood vessel's location will further advance minimally invasive liver surgery. Future research should focus on combining and overlapping the MRI and the laparoscope for more effective surgical procedures

To shorten setting time as can as possible, new type cylinder and new set method is advisable.

This study was partly supported by the Research for the Future Program (JSPS-RFTF 99I00904) Grant-in-Aid for Scientific Research (A) (14702070). NH was supported by Toyota Phsical\&Chemical Research Institute, Suzuken Memorial Foundation, Kurata Grants.

\section{References}

1. Masamune K., Kobayashi E., et al.: Development of an MRI compatible Needle Insertion Manipulator for Stereotactic Neurosurgery, J image Guided Surgery,1pp.242-248(1995)

2. Chinzei K., et al.: Surgical Assist Robot for the Active Navigation in the intraoperative MRI, MICCAI Proc.pp.921-930(2000)

3. Chinzei K., Hata N., et al.: MRcompatible Surgical Assist Robot: System Integration and Preliminary Feasibility Study, IEEE/RSJ IROS2000,Proc. 727-732(2000)

4. Robert B.Lufkin: Interventional MRI, pp.55-69, Mosby, (1999)

5. Osada A. et al.: The fundamental Investigation for the designing of MRI compatible instruments, JSCAS Proc.pp107-108 (2000)

6. Ikuda K., Ichikawa H., et al.: Study on Micro Pneumatic Drive for Safety Active Catheter, JSCAS Proc.pp99-100 (2001)

7. M.laseroms al.: A Hydraulic Forceps with Force-feedback for Use in Minimally Invasive Surgery, Mechatronics Vol.6, No 4, pp.437-446 (1996)

8. Saitou Y.,Sunagawa Y.; A study of Hydraulic Bilateral Servo Actuator for welfare Robots, Life Support Proc.pp100 (1998)

9. Bishoff, J.T., Stoianovici, D., Lee, B.R., Bauer, J., Taylor, R.H., Whitcomb, L.L., Cadeddu, J.A., Chan, D., Kavoussi, L.R., RCM-PAKY: Clinical Application of a New Robotic System for Precise Needle Placement, Journal of Endourology, Vol. 12, pp. S82 (1998)

10. Davies BL.: A Discussion of safety issues of medical robots, Computer integrated Surgery: Technology and Clinical Applications. Cambridge, MA: The MIT press, pp287-296 (1995) 